\begin{tabular}{lllll}
\multicolumn{4}{l}{ Abstract G244 } & Table 1 \\
\hline ST1-3 & ST4-6 & ST6+ & Consultant & Total \\
\hline 41 & 47 & 23 & 18 & 129 \\
$(31.78 \%)$ & $(36.4 \%)$ & $(17.82 \%)$ & $(13.95 \%)$ & \\
\hline
\end{tabular}

None of the responders answered all the questions correctly. There were wide discrepancies between objective and subjective knowledge of several aspects of ECGs.

Subjective interpretation of ECG axis scored the lowest with none of the level 1 doctors stating that they were confident; objective scores were better for this group at $21.8 \%$. The highest scores in the objective section were for calculating rates (100\% in the level 2 group).

Only $69 \%$ of the level 1 doctors said they were confident in lead placement against $87 \%$ of the consultants. Level 1 doctors scored only $51 \%$ in objective questions dealing with lead placement.

Confidence in interpretation of common ECG abnormalities was low with level 1 doctors at 5\% and consultants at 36\%. Objective scores scored better. ECGs on myocarditis, SVT and heart block scored better over pre-excitation, axis deviation and hypertrophy.

ECG showing Q waves were correctly identified by only 3 responders. Only $34 \%$ of the responders identified QTC interval as abnormal.

Responses were not always consistent with level of training and hade wide variations.

93\% of doctors responded favourably to an e-module for paediatric ECGs.Frequent requests for topics included age related changes, neonatal ECGs, tachyarrythmias, axis and QT interval interpretation.

Conclusions This survey revealed gaps and variations in ECG interpretation skills among paediatric doctors. Majority felt that an online problem based e-module on paediatric ECG interpretation would help in improving knowledge and confidence. This will be developed and uploaded to the e-learning for health care(ww.e-lfh.org.uk)website.

\section{G245 RISK FACTORS FOR ABNORMAL DEVELOPMENT AND COGNITIVE FUNCTION IN CHILDREN WITH CONGENITAL HEART DISEASE AND IMPLICATIONS FOR SERVICE PROVISION IN THE UK}

${ }^{1} \mathrm{~V}$ Banks, ${ }^{1} \mathrm{~A}$ Hoskote, ${ }^{1} \mathrm{~S}$ Kakat, ${ }^{2} \mathrm{D}$ Rideout, ${ }^{2} \mathrm{M}$ Lakhanpaul, ${ }^{2} \mathrm{C}$ Pagel, ${ }^{3} \mathrm{R}$ Franklin,
${ }^{4} \mathrm{~T}$ Witter. ${ }^{1}$ Cardiorespiratory Unit, Great Ormond Street Hospital, London, UK; ${ }^{2}$ University
College London, London, UK; ${ }^{3}$ Royal Brompton Hospital, London, UK; ${ }^{4}$ Evelina Childrens
Hospital, London, UK

10.1136/archdischild-2018-rcpch.238

Background Within the NHS context there is currently no specific additional neurodevelopmental follow up for children with congenital heart disease (CHD). Cardiologists are not trained in neurodevelopment and face many competing pressures, which may result in delayed detection of problems. We aimed to assess whether there is appropriate service provision for children with CHD and abnormal developmental outcome in the UK, and identify risk factors that could trigger early referral and more timely intervention.

Methods Usingvalidated scales of developmental/cognitive function with children less than 17 years of age with CHD treated at one of 3 centres in London, we assessed whether those with abnormal scores (defined as $>2$ standard deviations below the mean) were under follow up by appropriate developmental services. Logistic regression was used to identify potential socio-economic and clinical risk factors for adverse developmental and cognitive outcomes.

Results Being under follow up by developmental services was strongly associated $(\mathrm{p}<0.001)$ with abnormal developmental/ cognitive score but over $50 \%$ of patients with abnormal scores were not under any appropriate services. Risk factors associated with abnormal development/cognitive scores differed across age groups. Greater severity of CHD was associated with adverse gross motor development. Socio-economic factors were linked to adverse neurodevelopmental outcomes in older children.

Conclusion We identified a large number of patients with abnormal development not currently under appropriate care services, highlighting a potential failure in the care pathway for these children. A number of risk factors for adverse neurodevelopment were identified which could provide useful insight for clinicians to ensure high risk patients are appropriately referred, ensuring more timely assessment and intervention to enhance future development and quality of life for these children.

\section{G246(P) CLOSING THE DUCTUS ARTERIOSUS IN PRETERM INFANTS. A REVIEW OF PRESENT TREATMENT STRATEGIES AND DEVELOPING A DISEASE STAGING PROTOCOL}

H Raychaudhuri, A Bahadur, M Alam, S Nayak, I Ali. Neonatology, Newham Hospital, Barts and London NHS Trust, London, UK

10.1136/archdischild-2018-rcpch.239

Background The ductus arteriosus (DA) is an important structure in foetal life. Closure of the DA is an essential part of postnatal adaptation. Closure of the DA is initiated by an increase in oxygen and changes in pulmonary and systemic blood pressure. In preterm infants, failure of DA closure after birth can be associated with an increased incidence of chronic lung disease (CLD), intraventricular haemorrhage (IVH) and necrotizing enterocolitis (NEC). Prostaglandin inhibition using indomethacin or ibuprofen is the standard strategy to close the DA. Surgical closure and interventional device closure of the DA are an alternative option. Appropriate timing for closing the duct still remains a debatable topic. Various staging methods have been proposed based on Echocardiographic and clinical parameters to help clinicians make a decision. This study aims at assessing the effect of closing a DA on the overall morbidity and mortality in preterm infants and further interrogating available evidence on the best practice and optimum time for closing the DA. We designed a PDA staging protocol based on available evidence which will help clinicians decide on closing the DA.

Methods We conducted a review of literature and results from 10 different papers were assessed and analysed for this study.

Conclusion Closure of the DA in the first few weeks of life may provide short-term benefits. The long-term effects of untreated PDA in extremely premature infants remain unclear. Significant changes in management have evolved in the recent years including early surgical ligation and transcutaneous device closure but the evidence to support these changes are 